\title{
Modifiable Coronary Artery Disease Risk Factors Among Airline Pilots
}

\author{
N. Haddad ${ }^{1, *}$ and R. Hamadeh ${ }^{2}$ \\ ${ }^{1}$ Gulf Air Medical Services, Kingdom of Bahrain and ${ }^{2}$ Arabian Gulf University, Kingdom of Bahrain
}

\begin{abstract}
Introduction: Coronary Artery Disease (CAD) continues to be the leading cause of morbidity and mortality in developed countries. It is of particular concern in the aviation industry since it can result in sudden incapacitation. Moreover, it is the leading cause of pilot grounding. The aim of this study was to determine the prevalence of modifiable CAD risk factors among Gulf air pilots in Bahrain.

Method: All the pilots attending the Gulf Air clinic for their routine medical (253 pilots) were asked to fill an anonymous self administered questionnaire and were examined for weight, height and blood pressure. In addition past medical history and laboratory results were recorded. The CAD risk status was assessed using the Framingham score sheets.

Results: The prevalence of hypertension and diabetes mellitus were low while half of the pilots were overweight or obese. As for the other CAD risk factors, smoking, high triglyceride levels, hypercholesterolemia, and not performing regular exercise the prevalence rates were $26.1 \%, 43.6 \%, 7.1 \%$ and $17.1 \%$, respectively. $6.3 \%$ of the pilots had a calculated CAD risk acceptable for their age while $8.1 \%$ and $85.6 \%$ had a higher and lower risk, respectively.

Conclusion: Many pilots were found to be having high rates of CAD risk factors. Well structured primary and secondary preventive programs for pilots like smoking cessation, modifying dietary habits and promoting regular exercise should be initiated. An evaluation of the recommended preventive programs should follow.
\end{abstract}

Key Words: Coronary artery diseases risk factors, pilots, Bahrain.

\section{INTRODUCTION}

Non communicable diseases are the leading cause of morbidity and mortality in Bahrain with circulatory diseases accounting for $19.9 \%$ of all deaths in 2005 [1]. Cardiovascular diseases in general and coronary artery diseases (CAD) in particular, have the potential of causing sudden cardiac death [2] which is defined as a natural death due to cardiac causes, heralded by abrupt loss of consciousness within one hour of the onset of acute symptoms [3].

Most of the CAD risk factors are modifiable such as raised blood pressure and levels of total cholesterol, low density lipoprotein (LDL) and triglyceride. In addition, obesity, diabetes mellitus, smoking and lack of exercise and low levels of high density lipoprotein (HDL) are also modifiable. These risk factors usually cluster in individuals and there is a high association between the number of risk factors and overall cardiovascular risk [4-7]. The tremendous changes in lifestyle in Bahrain over the last five decades have resulted in a high prevalence of those risk factors. It has been reported that the prevalence of current smoking is $17.0 \%$; overweight, $35.2 \%$; obesity, $21.2 \%$, diabetes, $25.0 \%$; hypertension, $28.8 \%$; and only $12.7 \%$ do regular exercise among the adult population [8].

Identification of these factors and primary prevention is of particular importance in some occupations such as public transport drivers, airline or sea pilots or those working in jobs were public safety is of concern.

\footnotetext{
*Address correspondence to this author at the Kingdom of Bahrain, Gulf Air, P.O. Box 138, Bahrain; Tel: 00973-39898666; Fax: 00973-17332501; E-mail: nazar.haddad@gulfair.com and nazarhd@hotmail.com
}

In aviation, $\mathrm{CAD}$ is of greater concern because of its potential to cause sudden in-flight incapacitation. Since the early ages of aviation, sudden in-flight incapacitation is an important factor affecting flight safety. This concern has governed the development of medical standards both for screening before issuing a medical license and before resumption of flying following any medical condition. CAD in aviation is of high importance for many reasons: firstly, it is a disease and needs to be addressed in both primary and secondary prevention. Secondly, the knowledge about CAD and CAD risk factors can influence the strategies for a pilot's routine medical examination. Thirdly, CAD is the leading cause of pilot grounding and loss of licensing. Lastly, the high chance of sudden incapacitation may risk the population safety. Since controlling modifiable CAD risk factors can significantly lower the prevalence of the disease [9] and as studies on the prevalence of CAD and its factors among pilots are scarce in the region [10], this study was conducted. It aimed to determine the prevalence of modifiable CAD risk factors and describe some characteristics of the high risk groups among Gulf Air pilots in Bahrain.

\section{METHODOLOGY}

Ethical approval of the study was obtained from the operation department as well as from the pilots themselves. A letter was sent to each pilot explaining the objectives of the study and giving assurance regarding the privacy of any information given.

Around $94 \%$ of the pilots do their routine medical examination at the Gulf Air Medical Clinic while the others go 
to private clinics. This examination is conducted once annually for those younger than the age of 40 years and every six months for the older.

All the pilots attending the clinic for their routine medical examination between January and December 2004 were asked to participate in the study. Data on age, marital status, smoking status and exercise were obtained from the pilot and medical records were used for recording history of hypertension, diabetes mellitus, and use of medication, total cholesterol, HDL, LDL, triglycerides and uric acid levels as recorded in the last medical examination. Blood pressure, height and weight were measured by a trained nurse. Each pilot was asked to rest for at least 10 minutes before measuring the blood pressure. Blood pressure measurement was postponed when the pilot gave history of coffee (tea) drinking or smoking or exercising just prior to entering the clinic. A manual sphygmomanometer, with proper cuff size for each pilot was used to measure blood pressure on left arm. Weight in kilograms and height in meters were measured using a calibrated electronic device followed by the calculation of the body mass index (BMI). A pilot was considered overweight when his BMI was between 25 and 30 and obese when it was greater than 30 . A smoker was defined as a pilot who at the time of the study smoked any tobacco product either daily or occasionally [11]. The pilot was considered to be exercising if he practiced any type of exercise a minimum of 20 minutes at least three times per week.

The CAD risk status was measured using the Framingham heart study predictor score sheets [12]. Those factors include age, total cholesterol, high density lipoprotein cholesterol, blood pressure, diabetes mellitus and smoking.

\section{RESULTS}

Of the 270 total registered pilots, 253 attended the clinic and were examined for CAD risk factors. The remaining pilots did not attend the Gulf Air clinic and had their examination in a private clinic. The age range of the pilots was 2660 years with a mean of 38.3 years. $47.8 \%$ of the pilots were between $26-35$ years, $27.7 \%$ between $36-45$ years old and the rest $(24.5 \%)$ were older. The majority $(85.4 \%)$ of the pilots was ever married and $14.6 \%$ were never married with Arabs constituting 64.8\%, Europeans 25.0\%, Asians $6.0 \%$ and Africans $4.2 \%$ (Table 1).

The prevalence of hypertension among the pilots was $4.0 \%$. The majority $(93.6 \%)$ had a systolic blood pressure between $100-140 \mathrm{mmHg}, 3.8 \%<99 \mathrm{mmHg}$ and only $2.6 \%$ $>141 \mathrm{mmHg}$. Moreover, the diastolic blood pressure of $98.3 \%$ was $\leq 90 \mathrm{mmHg}$ and only $1.7 \%$ above that. Only four pilots were diabetic, three of whom used an oral hypoglycemic agent and one was on conservative treatment only.

Half of the pilots were ever smokers and $26.1 \%$ current smokers of any type of tobacco. $92.4 \%$ of the current smokers smoked cigarettes only, $7.6 \%$ sheesha (waterpipe) only, $4.6 \%$ cigar only and $10.7 \%$ were mixed smokers (cigarettes and sheesha). The prevalence of smoking was higher among pilots who were Arab, ever married and exercised. However, the prevalence of smoking among normal weight pilots was similar to that of overweight and obese pilots (Table 2 ).

$64.9 \%$ of the pilots had a cholesterol level below $200 \mathrm{mg} / \mathrm{dl}, 28.1 \%$ between $201-240 \mathrm{mg} / \mathrm{dl}$ and $7 \%$ above
$241 \mathrm{mg} / \mathrm{dl}$. The prevalence of hypercholesterolemia was found to be higher among Arabs, ever married, overweight and obese, smokers and those with low level of exercise with none of the relationships having statistical significance (Table 2). In addition $82.0 \%$ of the study population had HDL levels $\geq 35 \mathrm{mg} / \mathrm{dl}$ and $18.0 \%$ equal or below $34 \mathrm{mg} / \mathrm{dl}$. Furthermore, $89.2 \%$ of the pilots had LDL levels less or equal to $160 \mathrm{mg} / \mathrm{dl}$ and $10.8 \%$ greater and $48.9 \%$ had elevated triglycerides levels. The triglycerides level was also found to be higher among non- Arabs, ever married, those having hypercholesterolemia and those who exercised. The uric acid level was normal in $89.4 \%$ of the pilots and high in $10.6 \%$.

Table 1. Demographic Characteristics of Airline Pilots in Bahrain

\begin{tabular}{|c|c|c|}
\hline & No. & $\%$ \\
\hline \multicolumn{3}{|l|}{ Age Group (years) } \\
\hline $26-30$ & 59 & 23.3 \\
\hline $31-35$ & 62 & 24.5 \\
\hline $36-40$ & 26 & 10.3 \\
\hline $41-45$ & 44 & 17.4 \\
\hline $46-50$ & 39 & 15.4 \\
\hline $51-55$ & 15 & 5.9 \\
\hline $56-60$ & 8 & 3.2 \\
\hline \multicolumn{3}{|l|}{ Marital Status } \\
\hline Never married & 37 & 14.6 \\
\hline Ever married & 216 & 85.4 \\
\hline \multicolumn{3}{|l|}{ Nationality } \\
\hline Arabs & 164 & 64.8 \\
\hline Europeans & 63 & 25 \\
\hline Asians & 15 & 6 \\
\hline Africans & 11 & 4.2 \\
\hline
\end{tabular}

$82.9 \%$ of the study participants exercised at least 3 or more times per week. On the other hand, $17.1 \%$ did not exercise or exercised less than 3 times per week. Jogging and exercising at the gym were the most prevalent types practiced. Arabs, ever married and overweight and obese were less likely to be involved in regular exercise (Table 2).

Around half of the pilots were overweight and $13.6 \%$ obese with a mean BMI of $26.4 \pm 3.43 \mathrm{~kg} / \mathrm{m}^{2}$. Obesity and overweight were positively associated with the cholesterol level and negatively with practicing exercise.

Estimating CAD risk status using the Framingham heart study prediction score sheets showed that $85.6 \%$ of the pilots had a calculated risk less than acceptable while $8.1 \%$ had a risk more than the acceptable risk for their age and the rest $(6.3 \%)$ had a risk equal to their acceptable risk. 
Table 2. CAD Risk Factors, Nationality, Marital Status, Exercises and Body Mass Index

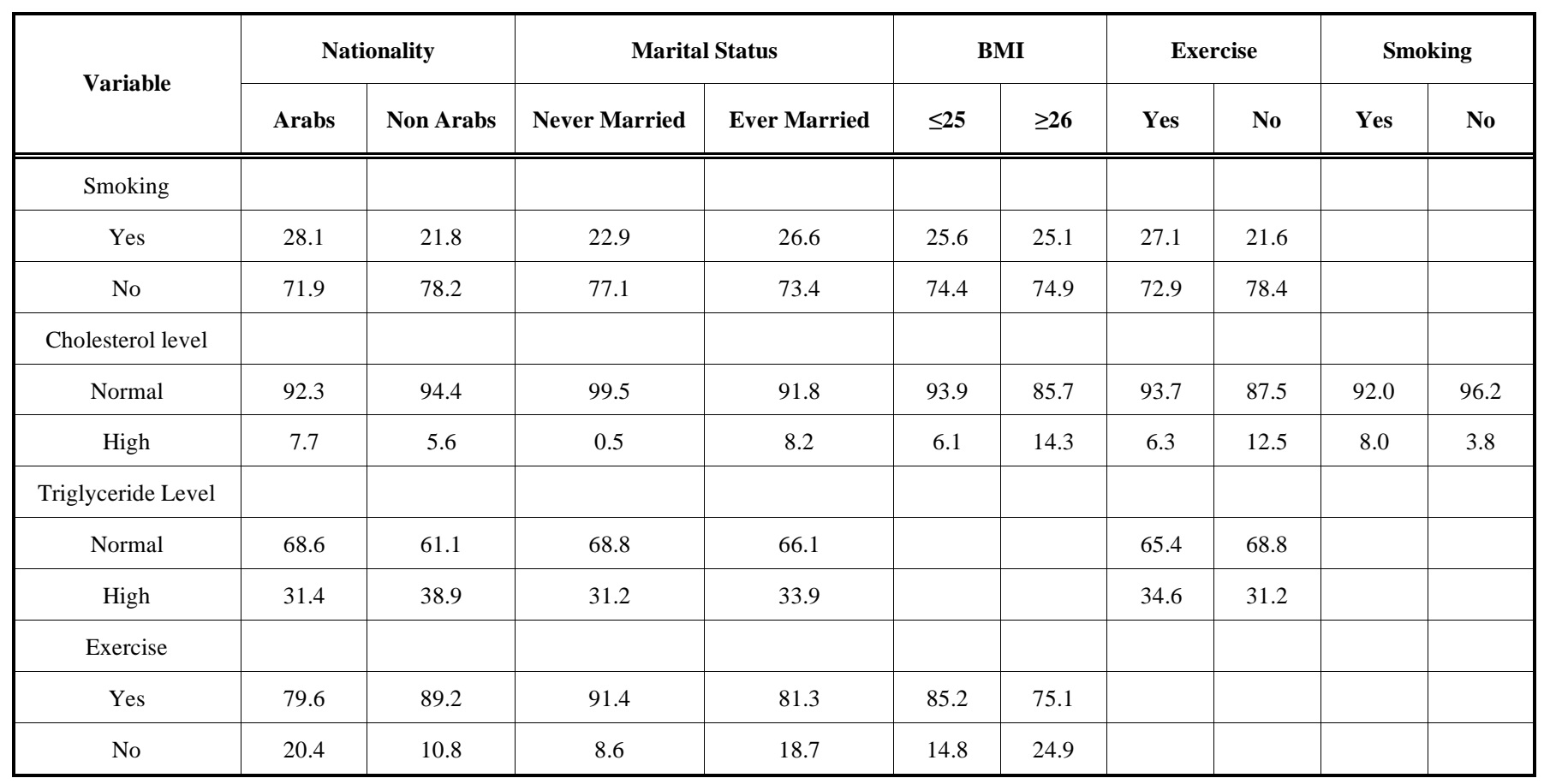

\section{DISCUSSION}

Airline pilots have to go through extensive medical assessment before they are appointed as trainees. Although there are certain health conditions that would be detected prior to employment and would disqualify the pilot on medical grounds from getting the license, other medical conditions may arise after he is considered qualified. If these conditions are detected, they will result in the grounding of the pilot and preventing him from flying thus creating a financial burden on both the airline company and the pilot himself. CAD is the leading cause of grounding pilots temporarily as well as permanently.

Several studies addressed the acceptable methods for CAD screening and identified the age it would be cost effective. The resting and exercise ECG were suggested as a suitable method for screening. However, a metaanalysis on 147 published reports of patients who underwent angiography and exercise testing [13] showed the sensitivity and specificity of exercise ECG for CAD detection as high as $68 \%$ and $77 \%$, respectively,. For issuing a class one (commercial pilot) medical certificate, the Joint Aviation Authorities requires a resting ECG for pilots. This investigation is required every 5 years, every 2 years, every year and every six months for those under 30 years of age, 30-39, 40- 50 and older, respectively. Exercise ECG is also required when there is a clinical indication such as chest pain, heart arrhythmias, hypertension and diabetes [14].

Primary prevention, especially when used for high risk groups could produce most cost effective long term benefits. With high selective criteria for pilot trainee, one should suspect very low CAD risk among young pilots, but afterwards the age factor increases the CAD risk. In many studies, a significant relationship was found between aging and cardiac risk $[10,15,16]$. A similar finding was noted in this study.
In this study, the prevalence rates of diabetes mellitus type 2 and hypertension were low and all cases were well controlled with one or two types of medication. This finding could be explained by the fact that most of the pilots were of the young age groups and both conditions are positively associated with age. In recent years, metabolic syndrome (diabetes mellitus or impaired glucose tolerance test with obesity) were associated with an increase risk of cardiovascular diseases [17]. Unfortunately, the pilots were not tested for glucose tolerance test was not done to the pilots and impaired glucose were not added as a risk factor for CAD which might have underestimated the CAD risk in this study.

Smoking prevalence was higher among younger age groups, Arabs and ever married pilots. This observation should be considered in any future primary prevention program. Smoking is a strong CAD risk factor and is considered over the other risk factors as the most common modifiable risk factor. Smoking has the advantage of being avoidable and when cessation occurs it results in a reduction in cardiovascular risk [18]. In this study, around one quarter of the participants was current smokers. This prevalence is comparable with that in the general adult male population in Bahrain [19]. This is surprising as one would expect a lower smoking rate among the pilots since in flight smoking is prohibited in the airlines. However, the lack of data on smoking prevalence among pilots before the implementation of the antismoking law makes it difficult to confirm if there has been a change in their smoking habits.

Epidemiological studies have clearly demonstrated the strong relationship between serum cholesterol levels and CAD risk [20]. At least $7 \%$ of the pilots had an elevated cholesterol level. The prevalence was lower than that reported in a Polish study where $72.4 \%$ were reported to have hypercholesterolemia [21]. Higher levels of serum cholesterol were 
found in Arabs and ever married respectively. A primary or secondary preventive program can make great difference in lowering serum lipid. In fact many studies have addressed the benefits of such programs in lowering serum lipids and preventing CAD [22-25].

Exercise, even in an individual without CAD risk, can significantly reduce the occurrence of CAD [26]. In addition, exercise has shown modest risk factor improvements in CAD patients [27]. The percentage of pilots performing regular exercise was relatively high and this observation should be encouraged and facilated more by the company. However, some subgroups like Arabs, ever married and overweight and obese pilots are less active than the others. Further studies are suggested to evaluate physical fitness of Gulf Air pilots.

Prospective studies of white men (like insurance cohort, airline pilots, cancer study volunteers and the Framingham population) have shown a positive linear relationship of weight to CAD [28]. In this study a high prevalence of overweight and obesity among pilots was found. This could be attributed to their sedentary lifestyle, lack of time for exercise, work stress and fatigue, many night shifts hours and unhealthy meals on the aircraft. The prevalence of overweight and obesity was similar to that reported from Poland (52.2\%) [21] but lower than in Australia (37.0\%) [23].

However, the prevalence rate over weight and obesity $(56.4 \%)$ among the pilots was similar to that of the general population in Bahrain.

It should be remembered that Framingham scores have some limitations when applied to any population [29]. Therefore; the calculated risk would not be very precise. Nevertheless it can be used for educational and future preventive strategies. Many earlier studies that have used the Framingham scores, or other types of scores, have concluded that the higher CAD scores, the more the likelihood to develop CAD $[22,30]$.

\section{CONCLUSION}

CAD remains the leading cause of morbidity and grounding among airline pilots. This study explored the CAD risk profile among Gulf Air Pilots in Bahrain. It was found that the pilot population has a high prevalence of some modifiable risk factors like smoking, overweight and obesity, hyperlipidemia, and lack of exercise but a low prevalence of diabetes mellitus and hypertension. According to the Framingham scores, around $8 \%$ would develop CAD within the coming 10 years.

Currently, there is no program in Bahrain that specifically targets high risk pilots in Gulf Air whether on the primary or secondary preventive levels. A well structured preventive program is urgently needed to cover all pilots, even those with a low CAD risk. This could be accomplished in collaboration with the national preventive programs in Bahrain.

Cardiac risk detection and modification should become an integral part of the routine medical examination of pilots. The investigations like exercise ECG should be added to their examination. Finally, further research on CAD risks in the Gulf Arab region should be considered.

\section{REFERENCES}

[1] Health information directorate, Ministry of Health. Health statistics 2005. Manama BH: Ministry of Health. Vital Statistics 2005; p.18.

[2] Saxon A. Sudden cardiac death: epidemiology and temporal trends. Rev Cardiovasc Med 2005; (Suppl 2): 512-20.

[3] Myerburg R, Castellanos A. Cardiac arrest and sudden cardiac death. In; Braunwald E, Zipes D, Libby P. Eds. Heart diseases: a Textbook of cardiovascular diseases. $6^{\text {th }}$ ed. Philadelphia: WB Saunders Co, 2001; 890-931.

[4] Campbell C, Nasir K, Blumenthal R. Metabolic syndrome, subclinical coronary atherosclerosis and cardiovascular risk. Am Heart Hosp J 2005; 3(2): 105-10.

[5] Meigs JB, D'Agostino RB Sr, Wilson PW, Cupples LA, Nathan $\mathrm{DM}$, Singer DE. Risk variable clustering in the insulin resistance syndrome. The Framingham Offspring study. Diabetes 1997; 46: 1504-600.

[6] Zanchetti A. The hypertensive patients with multiple risk factors: is treatment really so difficult? Am J Hyper 1997; 10: 223-9.

[7] Stamler J, Wentworth D, Neaton JD. Is relationship between cholesterol and risk of premature death from coronary artery disease continuous and graded? Findings in 256,222 primary screeners of the Multiple Risk Factors Intervention Trial (MRFIT). JAMA 1986; 256: 2823-8.

[8] Hamadeh R. Risk factors of major non communicable disease in Bahrain, the needs for surveillance system. Saudi Med J 2004; 25(9): 1147-52.

[9] Goldman L, Phillips KA, Coxson P, et al. The effect of risk factor reductions between 1981 and 1990 on coronary heart disease incidence, prevalence, mortality and cost. J Am Coll Cardiol 2001; 38: 1012-7.

[10] Tanej M, Wiegmann D. Prevalence of cardiovascular abnormalities in pilots involved in fatal general aviation airplane accidents. Aviation Space Environ Med 2002; 73(10): 1025-30.

[11] WHO, Guidelines for controlling and monitoring the tobacco epidemic. Geneva:WHO, 1997.

[12] Wilson P, D’Angostino R, Levy D, Belanger A, Silbershatz H, Kannel W. Prediction of coronary heart disease using risk factor categories. Circulation 1998; 97: 1837-47.

[13] Gianrossi R, Detrano R, Mulvihil D, et al. Exercise increase ST depression in the diagnosis of coronary artery disease. Circulation 1989; 80: 87

[14] Joint Aviation Requirements. JAR-FCL 3, flight crew licensing (medical). Class one medical requirement, amendment 1, 1-B-1. Global Engineering Documents, 15 Invernesse Way East, Englwood, Colorado 80112, USA, 2000.

[15] Holberg A. Cardiovascular diseases among U.S. Navy pilots. Aviation Space Environ Med 1985; 56: 397-402.

[16] Osswald S, Nixon W, Celio P. Review of cardiac events in USAF aviators. Aviation Space Environ Med 1996; 67: 1023-7.

[17] Eckle R, Grundy S, Zimmet P. The metabolic syndrome. Lancet 2005; 365: 1415-28.

[18] Central Statistics Organization. Basic Results Population, Housing, Buildings and Establishments Census, part one. Bahrain: Central Statistics Organization, Ministry of Cabinet Affairs, Central Statistics Organization, 2002.

[19] Critchley J, Capewell S. Smoking cessation for the secondary prevention of coronary heart diseases. Cochrane Database Syst Rev 2003; 4: CD003041.

[20] Mazurek K, Wielyosz A, Efenberg B, Orzel A. Cardivascular factors in supersonic pilots in Poland. Aviation Space Environ Med 2000; 71(12): 1202-5.

[21] Department of health and human services. Framingham heart study related meta-analysis of effect of LDL cholesterol 2007.

[22] Nacher E, Colangelo L, Beam C, Greenland P. Risk factors for coronary heart disease in men 18 to 39 years of age. Ann Intern Med 2002; 136(8): 631-2.

[23] Peal G, Booth M. Impact evaluation of the royal Australian air force health promotion program. Aviation Space Environ Med 2001; 72(1): 44-51.

[24] Bruse S, Grove S. The effect of a coronary risk evaluation program on serum lipid values and cardiovascular risk levels. Appl Nurs Res 1994; 7(2): 67-74.

[25] Multiple Risk Factor Intervention Trial Research Group. Mortality rates after 10.5 years for participants in the multiple risk factor intervention trial. Findings related to a priori hypothesis of the trial. JAMA 1990; 264(12): 1534-5. 
[26] Knutsen R. The tromso survey: The family intervention study- the effect of intervention on some coronary risk factors and dietary habits, 6 years follow up. Prev Med 1991; 20(2): 197-212.

[27] Brochu M, Poehlman E, Savage P, Fragnnoli-Munn K, Ross S, Ades PA. Modest effect of exercise training alone on coronary risk factors and body composition in coronary patients. J Cardiopulm Rehabil 2000; 20 (3): 180-8.

[28] Counor B. Obesity, arthrosclerosis and coronary artery disease. Ann Intern Med 1985; 103(62): 1010-9.
[29] Wilson P, D’Angiostino R, Levy D, Belanger A, Silbershatz H, Kannel W. Prediction of coronary heart disease using risk factors categories. Circulation 1998; 97: 1837-47.

[30] Clark D, Tolan G, Johnson R, Hickman J, Jackson W, McGranaham G. The west point study: 40 years of follow up. Aviation Space Environ Med 1994; 659(5): A71-4.

Received: October 31, 2007

Revised: May 4, 2008

Accepted: July 3, 2008

(C) Haddad and Hamadeh; Licensee Bentham Open.

This is an open access article licensed under the terms of the Creative Commons Attribution Non-Commercial License (http://creativecommons.org/licenses/ by-nc/3.0/) which permits unrestricted, non-commercial use, distribution and reproduction in any medium, provided the work is properly cited. 